\title{
Associated production of a single heavy $T$-quark in the littlest and simplest little Higgs models
}

\author{
Kingman Cheung ${ }^{1,2}$, C.S. Kim ${ }^{3}$, Kang Young Lee ${ }^{4}$, and Jeonghyeon Song ${ }^{5}$ \\ ${ }^{1}$ Department of Physics, National Tsing Hua University, Hsinchu, Taiwan R.O.C. \\ ${ }^{2}$ National Center for Theoretical Sciences, Hsinchu, Taiwan R.O.C. \\ ${ }^{3}$ Department of Physics, Yonsei University, Seoul 120-749, Korea \\ ${ }^{4}$ Department of Physics, KAIST, Daejeon 305-701, Korea \\ ${ }^{5}$ Department of Physics, Konkuk University, Seoul 143-701, Korea
}

(Dated: June 16, 2018)

\begin{abstract}
The colored $S U(2)_{L}$-singlet heavy $T$-quark is one of the most crucial ingredients in little Higgs models, which is introduced to cancel the largest contribution of the SM top quark to the Higgs boson mass at one-loop level. In two representative little Higgs models, the littlest Higgs model and the $S U(3)$ simplest Higgs model, we comprehensively study the single heavy $T$-quark production at Large Hadron Collider (LHC). After presenting the possibility of relatively light $(\sim 500 \mathrm{GeV})$ $T$-quark in the simplest little Higgs model, we consider all the relevant processes, the $2 \rightarrow 2$ process of $q b \rightarrow q^{\prime} T$, the $2 \rightarrow 3$ process of $q g \rightarrow q^{\prime} T \bar{b}$, the $s$-channel process of $q \bar{q}^{\prime} \rightarrow T \bar{b}$, and the gluon fusion process of $g g \rightarrow T \bar{t}$. We found that the $2 \rightarrow 3$ process can be quite important, as its cross section is about $30 \%$ of the $2 \rightarrow 2$ one and it is dominant in high $p_{T}$ distributions. The $s$-channel and the gluon fusion processes also show distinctive features in spite of their suppressed cross sections. In the gluon fusion process of the simplest little Higgs model, for example, the pseudo-scalar contribution is rather dominant over the Higgs contribution for relatively light $M_{T}$.
\end{abstract}

PACS numbers: 


\section{SINGLE TOP PRODUCTION}

Recently, new models, dubbed the "little Higgs" models, have drawn a lot of interests as new candidates for the solution of the gauge hierarchy problem [1]. The original idea dates back to 1970's: The lightness of the Higgs boson is attributed to its being a pseudo NambuGoldstone boson, which is generated when a global symmetry is spontaneously broken [2]. Unfortunately, the model was not phenomenologically viable due to the same quadratic divergence of the radiative correction to the Higgs mass as in the standard model (SM). Rearmed by the collective symmetry breaking idea, little Higgs models could explain the little hierarchy problem since the radiative generation of Higgs mass occurs at two-loop level. The one-loop level quadratic divergences from the SM gauge boson and top-quark loops are cancelled by those from new heavy gauge boson and heavy $T$-quark loops, respectively. Note that the cancellation at one-loop level happens between the SM particles and the new particles with the same statistics in the little Higgs model, unlike in supersymmetric theories. The exactly opposite coupling strength leads to the cancellation. This one-loop level cancellation is called the little Higgs mechanism.

Although there are many specific little Higgs models according to the global symmetry breaking pattern [3], we can classify them into two categories [4]. The first one is called the "product group" models, in which the diagonal breaking of two (or more) gauge groups leads to the SM gauge group. The most representative one is the littlest Higgs model. The second one is the "simple group" models, where a single gauge group is broken into the SM $S U(2)_{L}$ gauge group. The $S U(3)$ simple group model, so called the simplest little Higgs model, is one of the most studied simple group models. In literatures, most of phenomenological and experimental studies on little Higgs models at the Large Hadron Collider (LHC) have been focused on the littlest Higgs model [5, 6, 7, 8].

A new colored heavy $T$-quark is one of the most crucial ingredients of little Higgs models. The T-quark was introduced to cancel the SM top-quark contribution to the Higgs boson mass, which is the largest due to its large Yukawa coupling. Moreover, this T-quark decay pattern shows one novel feature of little Higgs models - the neutral decays of $T \rightarrow Z t$ and $T \rightarrow H t$ have equal branching ratios [9]. The first experimental signature of little Higgs models at high energy colliders would be the production of new heavy particles. However, the $T$-quark is considered to be quite heavy because of the strong constraint from the 
electroweak precision data (EWPD): The $T$-quark should be heavier than about one TeV, which suppresses its pair production at LHC. Naively estimated reach in a single $T$-quark production channel is up to $\sqrt{\hat{s}}$, while the reach in pair production is at most $\sqrt{\hat{s}} / 2$. Previous studies on the single $T$-quark production were, however, only on the total cross section of $q b \rightarrow q^{\prime} T$ mediated by the SM $W$ gauge boson [7]. Even though this $2 \rightarrow 2$ process has dominant total cross section, its $p_{T}$ distribution is prone to low $p_{T}$ region, compared to $2 \rightarrow 3$ process of $q g \rightarrow q^{\prime} T \bar{b}$. Also, the $s$-channel process of $q \bar{q}^{\prime} \rightarrow T \bar{b}$ can be quite sizable if the resonant decay of the heavy charged gauge boson is allowed.

Another process of interest is the gluon fusion with neutral scalar exchanges, which gives rise to heavy $T$-quark production associated with the SM top quark. It could be significant if the $T$-quark mass is light enough. As shall be discussed, the $T$-quark mass in the simplest little Higgs model can be considerably light at large $t_{\beta}$, where $t_{\beta}$ is the ratio of two vacuum condensates. In addition to the SM Higgs boson, the relatively light pseudo-scalar $\eta$ in the simplest little Higgs model can also mediate the gluon fusion process. We shall show that the contribution from $\eta$ exchange can dominate at small $M_{T}$.

Various kinematic distributions are also of great interest since the data-selection at LHC environment prefers high transverse momentum. We expect that the subdominant processes of the $2 \rightarrow 3$, the $s$-channel process, and the gluon fusion will become dominant in high $p_{T}$ region. Therefore, the main goal of the paper is to study comprehensively the single heavy $T$ quark production at LHC, including kinematic distributions, for both representative models, the littlest Higgs model and the $S U(3)$ simplest little Higgs model.

This paper is organized as follows. In Section III we review the basic formalism of the littlest Higgs model and the $S U(3)$ simplest little Higgs model. We explore the parameter space where the new heavy $T$-quark can be relatively light, and show that the $T$-quark mass in the simplest little Higgs model can be as low as about 500-700 GeV. Section III deals with the $T$-quark production accompanied by light quarks (including $b$ quark) without resort to the effective- $W$ approximation. We present the formula of the parton-level cross section for each process. In Section IV] we consider, focusing on the simplest little Higgs model, the gluon fusion process of $T t$ production mediated by the SM Higgs boson and the light pseudo-scalar boson $\eta$. Section $\nabla$ presents the numerical results of the total cross sections and various distributions. We summarize in Section VI 


\section{REVIEW OF THE LITTLEST HIGGS MODEL AND THE SIMPLEST LITTLE HIGGS MODEL}

\section{A. The littlest Higgs model}

The littlest Higgs model is embedded into a non-linear $\sigma$ model in the coset space of $S U(5) / S O(5)$ with additional local gauge symmetry $[S U(2) \otimes U(1)]^{2}[10,11]$. The covariant two-derivative term for the sigma field $\Sigma$ has the leading term of

$$
\mathcal{L}_{\Sigma}=\frac{1}{2} \frac{f^{2}}{4} \operatorname{Tr}\left|\mathcal{D}_{\mu} \Sigma\right|^{2}
$$

where the covariant derivative is

$$
\mathcal{D}_{\mu} \Sigma=\partial_{\mu} \Sigma+i \sum_{j=1}^{2}\left[g_{j} W_{j}^{a}\left(Q_{j}^{a} \Sigma+\Sigma Q_{j}^{a T}\right)+g_{j}^{\prime} B_{j}\left(Y_{j} \Sigma+\Sigma Y_{j}^{T}\right)\right] .
$$

At the scale $\Lambda_{S} \sim 4 \pi f$, a symmetric tensor of the $S U(5)$ global symmetry develops a vacuum expectation value $(\mathrm{VEV}) f \sim 1 \mathrm{TeV}$, of which direction is into the $\Sigma_{0}$ :

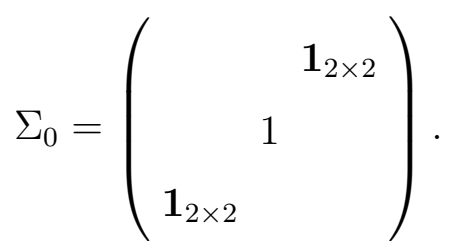

The $\Sigma_{0}$ triggers two kinds of symmetry breaking. First the global $S U(5)$ symmetry is broken into $S O(5)$, resulting in 14 massless Nambu-Goldstone bosons. Second, the assumed gauge symmetry $[S U(2) \otimes U(1)]^{2}$ is also broken into its diagonal subgroup $S U(2)_{L} \otimes U(1)_{Y}$,

identified as the SM gauge group. The gauge fields $\vec{W}^{\prime} \mu$ and $B^{\prime} \mu$ with the broken gauge symmetries become massive by eating four Nambu-Goldstone bosons.

The non-linear sigma fields are then parameterized by the Goldstone fluctuations:

$$
\Sigma=\Sigma_{0}+\frac{2 i}{f}\left(\begin{array}{ccc}
\phi^{\dagger} & \frac{h^{\dagger}}{\sqrt{2}} & \mathbf{0}_{2 \times 2} \\
\frac{h^{*}}{\sqrt{2}} & 0 & \frac{h}{\sqrt{2}} \\
\mathbf{0}_{2 \times 2} & \frac{h^{T}}{\sqrt{2}} & \phi
\end{array}\right)+\mathcal{O}\left(\frac{1}{f^{2}}\right)
$$

where $h$ is a doublet and $\phi$ is a triplet under the unbroken $S U(2)_{L}$. The gauge fields $\vec{W}^{\prime} \mu$ and $B^{\prime \mu}$ are related with the SM gauge fields by

$$
\begin{aligned}
W^{\mu} & =-s W_{1}^{\mu}-c W_{2}^{\mu}, & W^{\prime \mu} & =c W_{1}^{\mu}-s W_{2}^{\mu}, \\
B^{\mu} & =s^{\prime} B_{1}^{\mu}+c^{\prime} B_{2}^{\mu}, & B^{\prime \mu} & =-c^{\prime} B_{1}^{\mu}+s^{\prime} B_{2}^{\mu},
\end{aligned}
$$


where $g=g_{1} s=g_{2} c$ and $g^{\prime}=g_{1}^{\prime} s^{\prime}=g_{2}^{\prime} c^{\prime}$.

In order to cancel the severe quadratic divergence from the top quark loop, the top quark sector is extended with at least one additional top-quark-like fermion with a heavy mass of the order of $f$. In a minimal extension, a pair of $S U(2)_{L}$-singlet but colored Weyl fermions $U_{L}$ and $U_{R}$ with electric charge $+2 / 3$ are introduced. The Yukawa term for the third generation quarks and the singlet $u_{3 R}$ is [7]:

$$
\mathcal{L}_{\text {top }}=-\frac{\lambda_{1}}{2} f \chi_{L i}^{\dagger} \epsilon_{i j k} \epsilon_{m n} \Sigma_{j m} \Sigma_{k n} u_{3 R}-\lambda_{2} f U_{L}^{\dagger} U_{R}+\text { h.c. },
$$

where $i, j, k$ run between 1 and $3, m, n=4,5$, and $\chi_{L}^{T}=\left(-i d_{3 L}, i u_{3 L}, U_{L}\right)$ [8]. The $\Sigma$ field VEV mixes the $u_{L, R}$ fields with the $U_{L, R}$ fields into one massive mass-eigenstate $T_{0}$ (with $\mathrm{TeV}$ scale mass) and one massless eigenstate $t_{0}$ at this stage:

$$
\begin{array}{ll}
t_{0 L}=u_{3 L}, & t_{0 R}=\frac{\lambda_{2} u_{3 R}-\lambda_{1} U_{R}}{\sqrt{\lambda_{1}^{2}+\lambda_{2}^{2}}}, \\
T_{0 L}=U_{L}, & T_{0 R}=\frac{\lambda_{1} u_{3 R}+\lambda_{2} U_{R}}{\sqrt{\lambda_{1}^{2}+\lambda_{2}^{2}}} .
\end{array}
$$

The electroweak symmetry breaking (EWSB) is triggered by the Higgs boson VEV $v$, radiatively generated at two-loop level. This Higgs VEV also induces small mixing between $t_{0}$ and $T_{0}$, leading to final mass eigenstates $t$ and $T$ :

$$
T=T_{0}-x_{\lambda} \frac{m_{t}}{M_{T}} t_{0}, \quad t=t_{0}+x_{\lambda} \frac{m_{t}}{M_{T}} T_{0}
$$

where $x_{\lambda}=\lambda_{1} / \lambda_{2}$. Through this mixing, we have non-zero $W-T$ - $b$ coupling, though suppressed by $\sim v / f$.

The phenomenology of the littlest Higgs model at high energy colliders depends on the following parameters ${ }^{1}$ :

$$
f, \quad c, \quad c^{\prime}, \quad x_{\lambda},
$$

where one of $\lambda_{1}$ and $\lambda_{2}$ is traded with the SM top-quark mass. It is well known that main contributions to the EWPD are proportional to $c^{2}$ or $\left(c^{2}-s^{2}\right)$. In the parameter space around $c \ll 1$ and $c^{\prime}=1 / \sqrt{2}$, new contributions are therefore suppressed: $f \sim 2 \mathrm{TeV}$ is allowed in the region of $c \in[0,0.5]$ and $c^{\prime} \in[0.62,0.73][12,13]$.

\footnotetext{
${ }^{1}$ Even though the Higgs triplet VEV $v^{\prime}$ is also a model parameter, its effect on the collider phenomenology is negligible due to its smallness from the condition of positive definite mass squared of the Higgs triplet.
} 


\section{B. $S U(3)$ simple group model}

Among four popular representatives of little Higgs models, the $S U(3)$ simplest little Higgs model has the lowest fine-tuning associated to electroweak symmetry breaking [14]. The theory has an $\left[S U(3) \times U(1)_{X}\right]^{2}$ global symmetry and its diagonal subgroup $S U(3) \times U(1)_{X}$ is gauged. The kinetic term in the non-linear sigma model is

$$
\mathcal{L}_{\Phi}=\sum_{i=1,2}\left|\left(\partial_{\mu}+i g A_{\mu}^{a} T^{a}-\frac{i g_{x}}{3} B_{\mu}^{x}\right) \Phi_{i}\right|^{2}
$$

where $\Phi_{1,2}$ are the complex $S U(3)$ triplet scalar fields, $T^{a}$ are the $S U(3)$ generators, and $A_{\mu}^{a}$ and $B_{\mu}$ are, respectively, the $S U(3)$ and $U(1)$ gauge fields. Contrary to the littlest Higgs model, the two gauge couplings, $g$ and $g_{x}$, are determined by the SM gauge couplings: $S U(3)$ gauge coupling $g$ is just the $\mathrm{SM} S U(2)_{L}$ gauge coupling and

$$
g_{x}=\frac{g^{\prime}}{\sqrt{1-t_{W}^{2} / 3}} \approx 1.05 g^{\prime} .
$$

When $\Phi_{1}$ and $\Phi_{2}$ develop VEV's of TeV scale $\left\langle\Phi_{1,2}\right\rangle=f_{1,2}$, two kinds of symmetry breaking occur. First, the global symmetry is spontaneously broken into its subgroup of $[S U(2) \times U(1)]^{2}$, giving rise to ten Nambu-Goldstone bosons. Second, the gauge symmetry $S U(3) \times U(1)_{X}$ is broken into the $\mathrm{SM} S U(2)_{L} \times U(1)_{Y}$, as five Nambu-Goldstone bosons are eaten. Five new gauge bosons with $\mathrm{TeV}$ scale masses appear, a $Z^{\prime}$ gauge boson (a linear combination of $A^{8}$ and $\left.B^{x}\right)$ and a complex $\mathrm{SU}(2)$ doublet $\left(Y^{0}, X^{-}\right)$. The surviving NambuGoldstone bosons of a complex $S U(2)_{L}$ doublet $h$ and a SM singlet $\eta$ are parameterized by

$$
\Theta=\frac{1}{f}\left[\left(\begin{array}{ccc}
0 & 0 & \\
0 & 0 & h \\
h^{\dagger} & 0
\end{array}\right)+\frac{\eta}{\sqrt{2}}\left(\begin{array}{lll}
1 & 0 & 0 \\
0 & 1 & 0 \\
0 & 0 & 1
\end{array}\right)\right]
$$

where $h=\left(h^{0}, h^{-}\right)^{T}$ and $f=\sqrt{f_{1}^{2}+f_{2}^{2}}$. In the non-linear sigma model, they are related with two scalar fields through

$$
\Phi_{1}=e^{i t_{\beta} \Theta}\left(\begin{array}{c}
0 \\
0 \\
f c_{\beta}
\end{array}\right), \quad \Phi_{2}=e^{-i \Theta / t_{\beta}}\left(\begin{array}{c}
0 \\
0 \\
f s_{\beta}
\end{array}\right),
$$

where $t_{\beta}=f_{2} / f_{1}, c_{\beta} \equiv \cos \beta$ and $s_{\beta} \equiv \sin \beta$. 
Due to the $S U(3)$ gauge symmetry, the SM fermions are promoted to a $S U(3)$ triplet $\chi_{L}=\left(u_{L}, d_{L}, i U_{L}\right)^{T}$. For the third generation quarks, the Yukawa interaction is

$$
\mathcal{L}_{\text {top }}=i \lambda_{1} U_{R 1}^{\dagger} \Phi_{1}^{\dagger} \chi_{L}+i \lambda_{2} U_{R 2}^{\dagger} \Phi_{2}^{\dagger} \chi_{L}+\text { h.c. }
$$

where $U_{R 1}$ and $U_{R 2}$ are two additional $S U(2)_{L}$-singlet quarks, and $i$ 's guarantee positive mass for fermions. For the first two generation quarks and all generation leptons, Yukawa couplings can be imposed differently due to their negligible effects on radiative corrections to the Higgs mass. In literatures, two versions for the fermion embedding are discussed, "universal" model [17] and "anomaly-free" model [18]. In the universal embedding, all three generations have identical quantum numbers. We have heavy up-type quarks and heavy neutrinos. In the "anomaly-free" embedding, anomaly-cancellation is required for easier UV completion; the quarks of the third generation and three generations of leptons are put into 3 representations of $S U(3)$, while the first two generation quarks are put into $\overline{\mathbf{3}}$ of $S U(3)$. We have heavy down-type quarks for the first two generations. Irrespective of the fermion embedding, these two heavy quarks have TeV scale masses of [4]

$$
M_{Q}=s_{\beta} \lambda_{Q} f
$$

where $\lambda_{Q}$ is the Yukawa coupling with $\Phi_{2}$.

Substituting the VEV's of $\Phi_{i}$ into Eq. (14), a linear combination of $U_{R 1}$ and $U_{R 2}$ is coupled to $U_{L}$, which generates a TeV-scale Dirac mass. Its orthogonal combination has no mass term at this stage, to be identified with the SM top quark. The mass eigenstates before the EWSB are then

$$
\begin{aligned}
& t_{0 L}=u_{3 L}, \quad t_{0 R}=\frac{-\lambda_{2} s_{\beta} u_{3 R}+\lambda_{1} c_{\beta} U_{R}}{\sqrt{\lambda_{1}^{2} c_{\beta}^{2}+\lambda_{2}^{2} s_{\beta}^{2}}}, \\
& T_{0 L}=U_{L}, \quad T_{0 R}=\frac{\lambda_{1} c_{\beta} u_{3 R}+\lambda_{2} s_{\beta} U_{R}}{\sqrt{\lambda_{1}^{2} c_{\beta}^{2}+\lambda_{2}^{2} s_{\beta}^{2}}} .
\end{aligned}
$$

Finally, the EWSB causes a small mixing between $t_{0}$ and $T_{0}$ :

$$
T=T_{0} \mp \frac{1}{\sqrt{2} t_{\beta}} \frac{v}{f} t_{0} \equiv T_{0}-\delta_{t} t_{0}, \quad t=t_{0} \pm \frac{1}{\sqrt{2} t_{\beta}} \frac{v}{f} T_{0},
$$

where the upper sign is for the anomaly-free model and the lower sign is for the universal model. 
TABLE I: Masse of heavy particles in the littlest and simplest little Higgs model. Here $x_{\lambda}=\lambda_{1} / \lambda_{2}$ and $t_{\beta}=f_{2} / f_{1}$.

\begin{tabular}{lll}
\hline \hline & littlest & simplest \\
\hline charged heavy gauge boson & $M_{W_{H}}=\frac{g f}{2 s c}$ & $M_{X^{ \pm}}=\frac{g f}{\sqrt{2}}$ \\
neutral heavy gauge boson & $M_{Z_{H}}=\frac{g f}{2 s c}$ & $M_{Y^{0}}=\frac{g f}{\sqrt{2}}$ \\
& $M_{B_{H}}=\frac{g^{\prime} f}{2 \sqrt{5} s^{\prime} c^{\prime}}$ & $M_{Z^{\prime}}=\sqrt{\frac{2}{1-t_{W}^{2}} g f}$ \\
heavy $T$-quark & $M_{T}=\left(x_{\lambda}+\frac{1}{x_{\lambda}}\right) \frac{m_{t}}{v} f$ & $M_{T}=\sqrt{2} \frac{t_{\beta}^{2}+x_{\lambda}^{2}}{\left(1+t_{\beta}^{2}\right) x_{\lambda}} \frac{m_{t}}{v} f$ \\
\hline \hline
\end{tabular}

Although different heavy fermion spectrum itself is very exciting, the single heavy $T$-quark production at LHC is not directly affected by other heavy fermions. Their only effect is the parameter space allowed by the EWPD in each model [15, 16]. According to Ref. [15], the universal model is strongly constrained by its new contributions to atomic parity violation, leading to $f \gtrsim 3.9 \mathrm{GeV}$ at $95 \%$ C.L., while the anomaly-free model is more sensitive to LEP II data, leading to $f \gtrsim 2 \mathrm{TeV}$. In addition, the contributions to EWPD are suppressed by large $t_{\beta}$. Thus, a large $t_{\beta}$ leads to interesting implications for heavy fermion spectra. As shall be shown below, heavy $T$-quark mass decreases with increasing $t_{\beta}$ while the first two generation heavy quark masses increase with $t_{\beta}$ as in Eq. (15). In what follows, therefore, we examine the parameter space of $f \geq 2 \mathrm{TeV}$ and large $t_{\beta}$ for the simplest little Higgs model.

\section{Heavy masses and relevant couplings}

In little Higgs models, the SM gauge boson masses are modified with corrections of the order of $v^{2} / f^{2}$. Their effects at LHC are, however, negligible if we only consider the parameter space allowed by EWPD. Masses of heavy particles in the littlest and simplest little Higgs models are summarized in Table 1

The dominant single $T$-quark production at LHC is accompanied by a light quark. The production of $T \bar{b}$ is mediated by the charged gauge boson $W$ and $W_{H}\left(X^{ \pm}\right)$in the littlest 
TABLE II: The charged current couplings for $W$ and $W_{H}$ or $X^{ \pm}$. In the simplest little Higgs model, the flavor misalignment is not assumed for simplicity, and the upper (lower) sign is for the anomaly-free (universal) embedding.

\begin{tabular}{lll}
\hline \hline & littlest & simplest \\
\hline$g_{u d}^{W}$ & $\frac{g}{\sqrt{2}}\left(1-\frac{\Delta}{2} c^{2}\left(c^{2}-s^{2}\right)\right)$ & $\frac{g}{\sqrt{2}}\left(1-\frac{\Delta}{4 t_{\beta}^{2}}\right)$ \\
$g_{u d}^{W^{\prime}}$ & $-\frac{g}{\sqrt{2}} \frac{c}{s}$ & $\pm \frac{g}{2 t_{\beta}} \frac{v}{f}$ \\
$g_{T b}^{W}$ & $x_{\lambda} \frac{g}{\sqrt{2}} \frac{m_{t}}{M_{T}}$ & $-\frac{g}{\sqrt{2}} \frac{t_{\beta}}{1+t_{\beta}^{2}}\left(x_{\lambda}-\frac{1}{x_{\lambda}}\right) \frac{m_{t}}{M_{T}}$ \\
$g_{T b}^{W^{\prime}}$ & $-x_{\lambda} \frac{g}{\sqrt{2}} \frac{m_{t}}{M_{T}} \frac{c}{s}$ & $\frac{g}{\sqrt{2}}$ \\
\hline \hline
\end{tabular}

(simplest) Higgs model. We denote the relevant couplings as

$$
\mathcal{L}=-\sum_{V=W, W^{\prime}} g_{u d}^{V} \bar{d} \gamma^{\mu} P_{L} u V_{\mu}-\sum_{V=W, W^{\prime}} g_{T b}^{V} \bar{T} \gamma^{\mu} P_{L} b V_{\mu} \quad+\text { h.c. }
$$

where $W^{\prime}=W_{H}$ in the littlest Higgs model and $W^{\prime}=X^{ \pm}$in the simplest little Higgs model. Here the Cabibbo-Kobayashi-Maskawa (CKM) matrix factors are omitted. In Table II we summarize the relevant couplings for the charged-currents with the $W$ and $W^{\prime}$ gauge bosons.

The mass of the heavy $T$-quark plays an important role in its production cross section. A relatively light $T$-quark helps reduce fine-tuning in the Higgs boson mass, as well as increasing the production rates for the single heavy $T$-quark. In the littlest Higgs model, the $M_{T}$ is invariant under $x_{\lambda} \leftrightarrow 1 / x_{\lambda}$, so that the minimum of $M_{T}$ occurs when $x_{\lambda}=1$ :

$$
M_{T}^{(\min )}=2 \frac{m_{t}}{v} f \approx 1.42 f \quad \text { in the littlest Higgs model. }
$$

With the allowed value of $f \gtrsim 1.5 \mathrm{TeV}$ by the EWPD, the heavy $T$-quark is quite heavy in the littlest Higgs model.

In the simplest little Higgs model, $M_{T}$ can be substantially less. The minimum of $M_{T}$ occurs when $x_{\lambda}=t_{\beta}$ :

$$
M_{T}^{(\min )}=2 \sqrt{2} \frac{t_{\beta}}{1+t_{\beta}^{2}} \frac{m_{t}}{v} f \quad \text { when } x_{\lambda}=t_{\beta} .
$$

In Fig. 1, we have plotted $M_{T} / f$, in the simplest little Higgs model, as a function of $x_{\lambda}$ for $t_{\beta}=1,2,7$. It is very interesting that $M_{T}$ can be significantly reduced for large $t_{\beta}$. For 


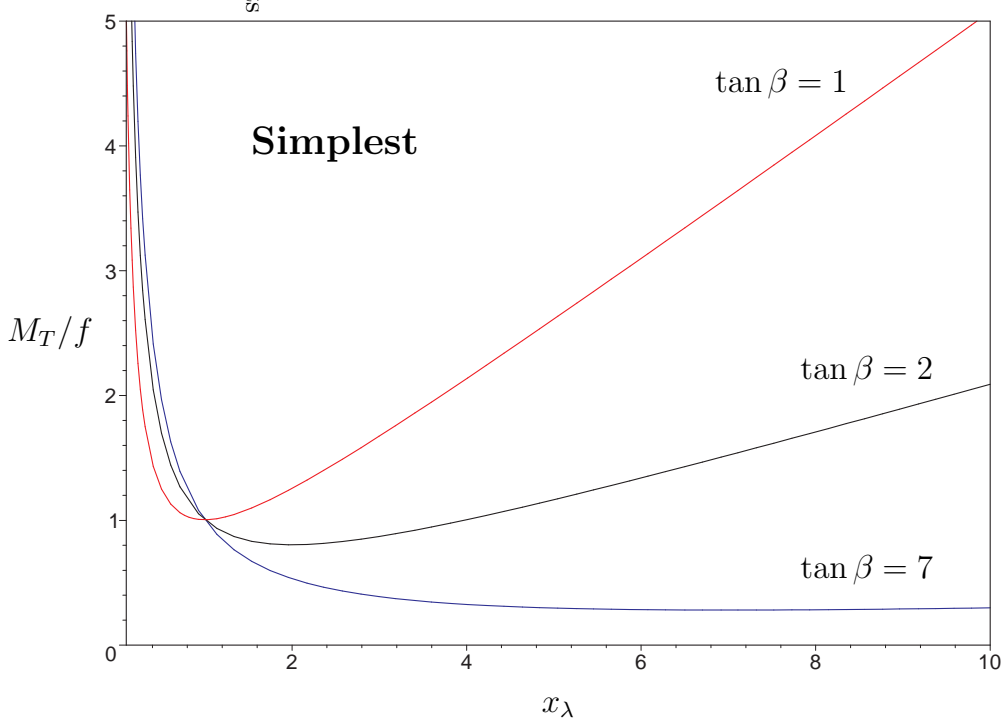

FIG. 1: The heavy $T$-quark mass in unit of $f$ as a function of $x_{\lambda}$ for $t_{\beta}=1,2,7$ in the $S U(3)$ simplest little Higgs model.

example, taking $x_{\lambda}=3(7)$ and $t_{\beta}=7$ leads to $M_{T}=0.39(0.28) f$ : Even for $f=2 \mathrm{TeV}$, the heavy $T$-quark mass is only $780(563) \mathrm{GeV}$, which is very accessible at LHC.

Another interesting region of parameter space is where the new charged gauge boson $W^{\prime}$ becomes heavier than the $T$-quark so that the resonant production of $u \bar{d} \rightarrow W^{\prime} \rightarrow T \bar{b}$ is kinematically allowed. Figure 2 shows the parameter space where the $W^{\prime}$ is heavier than the $T$-quark. In the littlest Higgs model, for example, the parameter space of $c<0.24$ $(c<0.19)$ for $x_{\lambda}=1\left(x_{\lambda}=2\right)$ guarantees the $W_{H}$ heavier than the $T$-quark. Unless $x_{\lambda}$ is too large, a small value of $c$ ensures the resonant decay of $W_{H}$ into a $T \bar{b}$ pair. Moreover, a small $c$ is preferred by the EWPD. Since the minimum of $M_{T}$ is more or less fixed in the littlest Higgs model, the condition of $M_{T}<M_{W_{H}}$ is satisfied by the heaviness of $W_{H}$ gauge boson, not by the lightness of the $T$-quark.

In the simplest little Higgs model, the mass of charged gauge boson $X^{ \pm}$is fixed at $g f / \sqrt{2}$. The $M_{T}$ depends on two parameters, $t_{\beta}$ and $x_{\lambda}$. As shown in Fig. 2(b), there are two regions in the parameter space for $M_{X}>M_{T}$ : One is where $x_{\lambda} \geq 2 m_{t} /(g v) \approx 2.18$ and $t_{\beta} \gtrsim 4$; the other is where both parameters are very small (note that it is barely visible from the figure near the origin). Considering the EWPD preference for larger $t_{\beta}$ as well as avoiding too small parameters, we take $t_{\beta}>1$. Consequently the resonant decay of $X^{ \pm}$requires large $t_{\beta}$. In summary, as $t_{\beta}$ increases the heavy $T$-quark becomes lighter, the EWPD constraints 

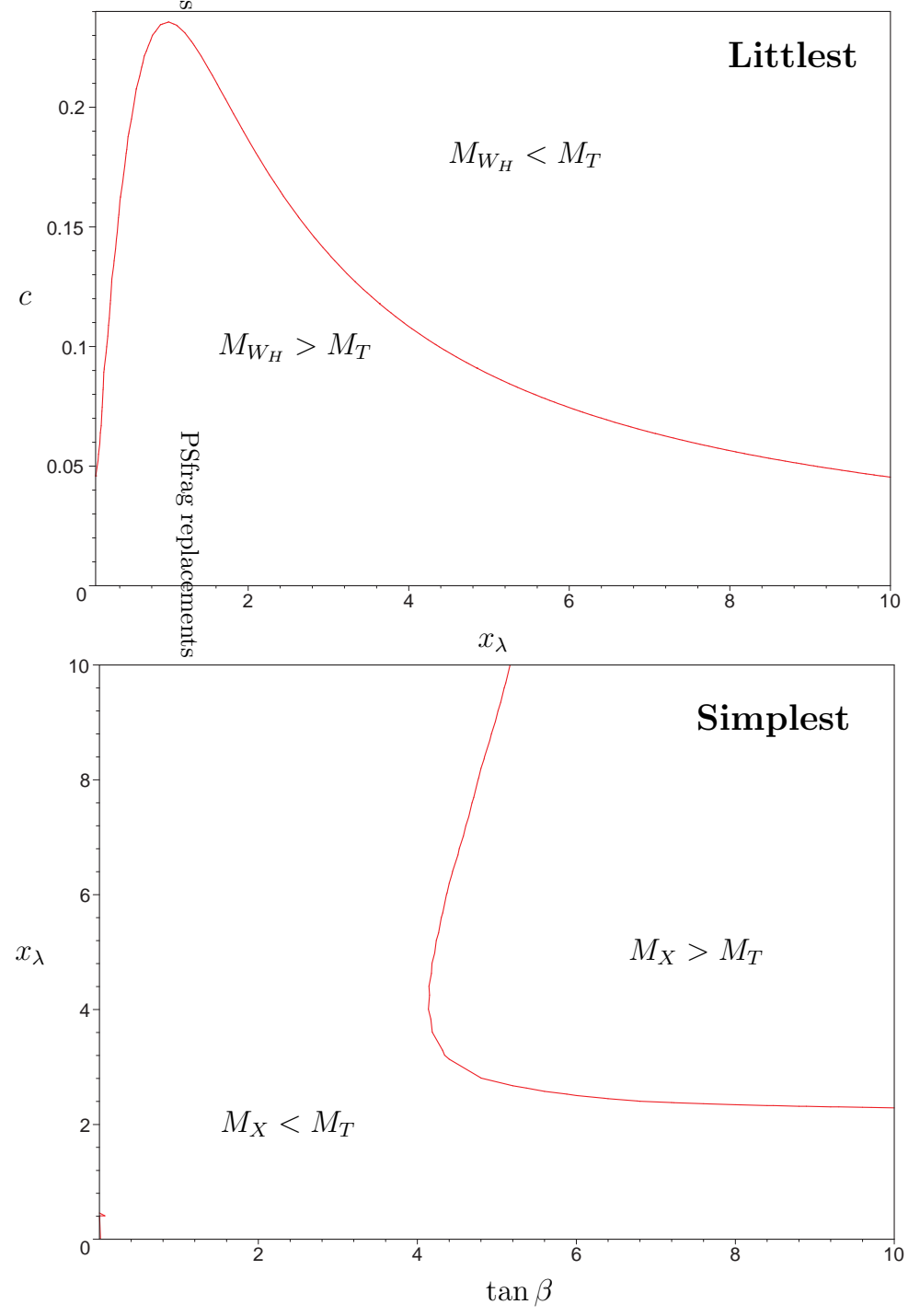

FIG. 2: The parameter space where $W^{\prime}$ is heavier than the $T$-quark in the (a) littlest and (b) simplest little Higgs models

become milder, and the couplings of $X-u-d$ and $W-T-b$ become smaller, while the $X-T-b$ coupling remains intact.

\section{THE SINGLE HEAVY T-QUARK PRODUCTION}

A heavy $T$-quark can be produced through Feynman diagrams in Fig. 3. We denote the cross section for diagram (a) by $\sigma_{2 \rightarrow 2}$, for diagram (b) by $\sigma_{2 \rightarrow 3}$, and for diagram (c) by $\sigma_{\mathrm{s}-\mathrm{ch}}$. 


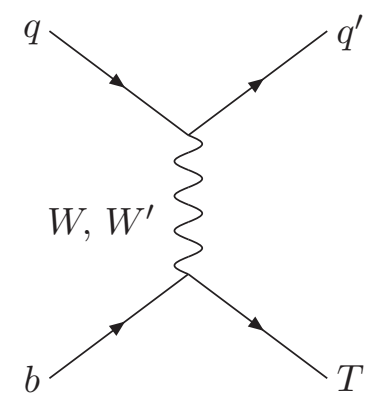

(a)

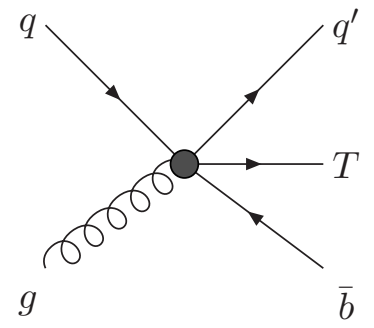

(b)

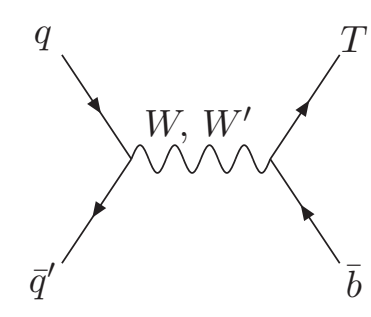

(c)

FIG. 3: Feynman diagrams for the heavy $T$-quark production at hadron colliders.

In general, the total cross sections from these channels show the following hierarchy:

$$
\sigma_{2 \rightarrow 2}>\sigma_{2 \rightarrow 3}>\sigma_{\mathrm{s}-\mathrm{ch}} .
$$

A possible collinear divergence in the $\sigma_{2 \rightarrow 3}$ [19] is avoided by applying kinematic cuts on the outgoing light quark. In literatures, only the $\sigma(W b \rightarrow T)$ is discussed [7]. However, the $t$-channel process of $\sigma_{2 \rightarrow 2}$ generates, in general, a low $p_{T}$ distribution. In high $p_{T}$ regime, the $2 \rightarrow 3$ process is expected to become important.

\section{A. Two-body production through $t$ channel diagram}

We study the heavy $T$-quark production associated with a single light quark through the following parton level processes:

$$
u(p)+b\left(p_{b}\right) \rightarrow d\left(p^{\prime}\right)+T\left(p_{T}\right), \quad \text { or } \quad \bar{d}(p)+b\left(p_{b}\right) \rightarrow \bar{u}\left(p^{\prime}\right)+T\left(p_{T}\right)
$$

For each process, there are two Feynman diagrams mediated by the $W$ and heavy $W^{\prime}$ gauge bosons as in Fig. 3(a). 
We define the Mandelstam variables as

$$
\hat{s}=\left(p+p_{b}\right)^{2}, \quad \hat{t}=\left(p-p^{\prime}\right)^{2}, \quad \hat{u}=\left(p_{b}-p^{\prime}\right)^{2} .
$$

The spin-averaged amplitude-squared at the parton-level are

$$
\begin{aligned}
& \overline{|M|^{2}}(u b \rightarrow T d)=\left|Q_{\hat{t}}\right|^{2} \frac{\hat{s}\left(\hat{s}-M_{T}^{2}\right)}{\left(\hat{t}-m_{W}^{2}\right)^{2}}, \\
& \overline{|M|^{2}}(\bar{d} b \rightarrow T \bar{u})=\left|Q_{\hat{t}}\right|^{2} \frac{\hat{u}\left(\hat{u}-M_{T}^{2}\right)}{\left(\hat{t}-m_{W}^{2}\right)^{2}},
\end{aligned}
$$

where

$$
Q_{\hat{t}}=g_{u d}^{W} g_{T b}^{W}+g_{u d}^{W^{\prime}} g_{T b}^{W^{\prime}} \frac{\hat{t}-m_{W}^{2}}{\hat{t}-M_{W^{\prime}}^{2}} .
$$

The couplings $g_{q q^{\prime}}^{W, W^{\prime}}$ are listed in Table The parton-level cross section is well known as

$$
\frac{d \hat{\sigma}}{d \cos \Theta}=\frac{1}{32 \pi \hat{s}}\left(1-\frac{M_{T}^{2}}{\hat{s}}\right) \overline{|M|^{2}}
$$

where $\Theta$ is the scattering angle of the heavy $T$-quark in the c.m. frame of partons. Note that all of the processes have color factors equal to one.

\section{B. Two-body process via $s$-channel diagram and $W^{\prime}$ decay rate}

For the single heavy $T$-quark production, there is also $s$-channel process of

$$
u\left(p_{1}\right)+\bar{d}\left(p_{2}\right) \rightarrow T\left(p_{T}\right)+\bar{b}\left(p_{\bar{b}}\right) .
$$

Due to the suppressed luminosity of the sea quark, the cross section is in general subdominant. Since this process is mediated by the heavy $W^{\prime}$ gauge boson as well as the $W$ gauge boson, however, a possible resonant mediation by the $W^{\prime}$ gauge boson can enhance its contribution.

The spin- and color-averaged amplitude squared is

$$
\overline{|M|^{2}}(u \bar{d} \rightarrow \bar{b} T)=\left|Q_{\hat{s}}\right|^{2} \frac{\hat{t}\left(\hat{t}-M_{T}^{2}\right)}{\left(\hat{s}-m_{W}\right)^{2}},
$$

where $\hat{s}=\left(p_{1}+p_{2}\right)^{2}, \hat{t}=\left(p_{1}-p_{\bar{b}}\right)^{2}$, and

$$
Q_{\hat{s}}=g_{u d}^{W} g_{T b}^{W}+g_{u d}^{W^{\prime}} g_{T b}^{W^{\prime}} \frac{\hat{s}-m_{W}^{2}}{\hat{s}-M_{W^{\prime}}^{2}+i M_{W^{\prime}} \Gamma_{W^{\prime}}} .
$$


Some discussions on the total decay rate of the heavy $W^{\prime}$ gauge boson are in order here. In the littlest Higgs model, $W_{H}$ can decay into $\mathrm{SM}$ fermion pairs or $W h$. In addition, if kinematically allowed, the $W_{H}$ can decay into $T \bar{b}$ as well. The total decay rate is

$$
\Gamma^{\mathrm{Lst}}\left(W_{H}\right)=\sum_{f} \Gamma\left(W_{H} \rightarrow f \bar{f}^{\prime}\right)+\Gamma\left(W_{H} \rightarrow W h\right)+\Gamma\left(W_{H} \rightarrow T \bar{b}\right) \theta\left(M_{W^{\prime}}-M_{T}-m_{b}\right)
$$

Partial decay rates are

$$
\begin{aligned}
\sum_{f} \Gamma\left(W_{H} \rightarrow f \bar{f}^{\prime}\right) & =\frac{g^{2} \cot ^{2} \theta}{4 \pi} M_{W_{H}}, \\
\Gamma\left(W_{H} \rightarrow W h\right) & =\frac{g^{2} \cot ^{2} 2 \theta}{192 \pi} M_{W_{H}} \\
\Gamma\left(W_{H} \rightarrow T \bar{b}\right) & =\frac{g^{2} x_{\lambda}^{2} \cot ^{2} \theta}{16 \pi}\left(\frac{m_{t}}{M_{T}}\right)^{2} M_{W_{H}}\left(1-\frac{M_{T}^{2}}{M_{W_{H}}^{2}}\right),
\end{aligned}
$$

where $\theta$ is the mixing angle in the gauge sector, defined by Eq. (15).

In the simplest little Higgs model, the $X^{ \pm}$gauge boson generically couples the new heavy quark to a SM quark. For simplicity, we assume that the first two generation heavy fermions $Q^{1,2}$ are heavy enough so that the $X^{ \pm} \rightarrow Q^{1,2} \bar{f}$ decay is kinematically prohibited. If $M_{X^{+}}>M_{T}$, the main decay mode is $X^{+} \rightarrow T \bar{b}$ with a partial decay rate of

$$
\Gamma^{S U(3)}(X \rightarrow T \bar{b})=\frac{g^{2}}{16 \pi} M_{X}\left(1-\frac{M_{T}^{2}}{M_{X}^{2}}\right) .
$$

When $X^{+} \rightarrow T \bar{b}$ is not kinematically allowed, the decay into the SM fermions $X^{+} \rightarrow f \bar{f}^{\prime}$ becomes dominant. The decay rate summed over the SM fermions is

$$
\sum_{f, f^{\prime}} \Gamma\left(X \rightarrow f \bar{f}^{\prime}\right)=\frac{g^{2}}{8 \pi t_{\beta}^{2}} \frac{v^{2}}{f^{2}} M_{X}
$$

which is suppressed by small $v^{2} / f^{2}$ and large $\tan \beta$. This is possible due to the small mixing between the SM fermion and the heavy fermion.

\section{Three-body production}

The heavy $T$-quark production through $q g \rightarrow T \bar{b} q^{\prime}$ can become dominant in the high $p_{T}$ region. Since we are interested in the kinematic distributions, we compute the differential cross section without resort to the effective- $W$ approximation. Instead, we consider the whole 


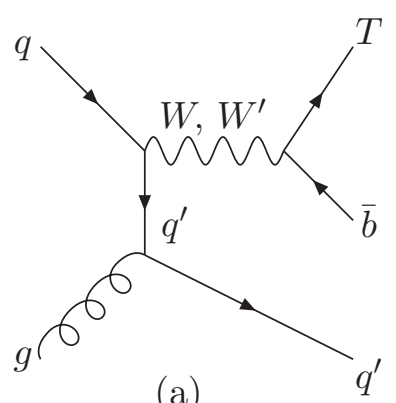

(a)

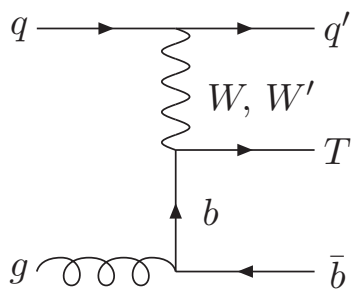

(c)

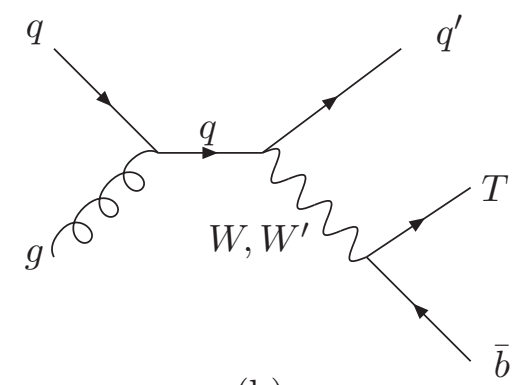

(b)

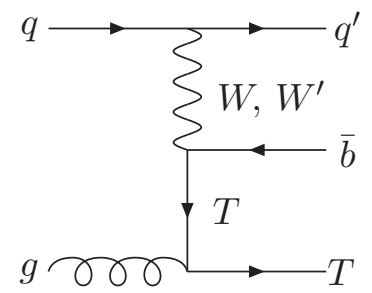

(d)

FIG. 4: Feynman diagrams for $g u \rightarrow d \bar{b} T$.

set of Feynman diagrams, mediated by $W$ and $W^{\prime}$ gauge bosons, as depicted in Fig. 4. For the process of

$$
u\left(p_{1}\right)+g\left(p_{2}\right) \rightarrow d\left(k_{1}\right)+T\left(k_{2}\right)+\bar{b}\left(k_{3}\right),
$$

the amplitude-squared (after summing over the final-state colors and helicities, and averaging over initial-state colors and helicities) is given by Eq. (A2) in the Appendix. The parton-level cross section is

$$
d \hat{\sigma}=\frac{1}{2^{10} \pi^{4}} \overline{|M|^{2}} \mathrm{~d} \eta \mathrm{d} \zeta \mathrm{d} \alpha \mathrm{d} \cos \beta,
$$

where the definitions and allowed range of kinematic variables $(\eta, \zeta, \alpha$, and $\beta$ ) are also referred to the Appendix.

\section{IV. $g g \rightarrow T \bar{t}$ MEDIATED BY SCALARS}

Due to the high c.m. energy of $14 \mathrm{TeV}$ at LHC, the gluon luminosity dominates over quark luminosity unless some very heavy particles are produced. As discussed before, the simplest little Higgs model can accommodate a relatively light $T$-quark with a mass as low as $600 \mathrm{GeV}$. In addition, the SM Higgs boson $H$ and the pseudoscalar $\eta$ couple the SM 


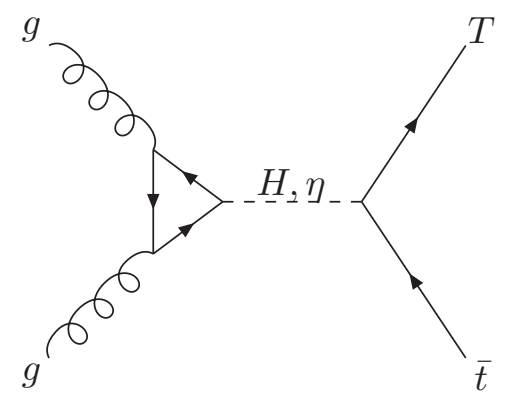

FIG. 5: Feynman diagrams for $g g \rightarrow T \bar{t}$.

top quark $t$ and the new heavy $T$-quark, through the mixing between $t$ and $T$ induced by the EWSB. Phenomenological impacts and signatures of the $\eta$ boson at the LHC and other future colliders have been discussed in Ref. [20]. In this section, we study in the simplest little Higgs model the gluon-fusion production of the heavy $T$-quark associated with $\bar{t}$ quark, as depicted in Fig. 5,

We parameterize the Yukawa terms as

$$
\mathcal{L}_{Y}=-\frac{m_{f}}{v} y_{f}^{H} \bar{f} f H-\frac{m_{f}}{v} y_{f}^{\eta} i \bar{f} \gamma_{5} f \eta+\kappa_{H}\left(H \bar{T} P_{L} t+h . c .\right)+\kappa_{\eta}\left(i \eta \bar{T} P_{R} t+\text { h.c. }\right),
$$

where $P_{R, L}=\left(1 \pm \gamma_{5}\right) / 2$, and

$$
\begin{aligned}
y_{t}^{H} & =1+\mathcal{O}\left(\frac{v^{2}}{f^{2}}\right), \quad y_{t}^{\eta}=\frac{\left[t_{\beta}^{4}+2 t_{\beta}^{2}\left(x_{\lambda}^{2}-1\right)-x_{\lambda}^{2}\right]}{\sqrt{2} t_{\beta}\left(t_{\beta}^{2}+x_{\lambda}^{2}\right)} \frac{v}{f}, \\
y_{T}^{H} & =-\frac{x_{\lambda}^{2}\left(t_{\beta}^{2}+1\right)}{2\left(t_{\beta}^{2}+x_{\lambda}^{2}\right)^{2}} \frac{v^{2}}{f^{2}}, \quad y_{T}^{\eta}=\frac{t_{\beta}\left(x_{\lambda}^{2}-1\right)}{\sqrt{2}\left(t_{\beta}^{2}+x_{\lambda}^{2}\right)} \frac{v}{f}, \\
\kappa_{H} & =\frac{t_{\beta}}{t_{\beta}^{2}+1} \frac{x_{\lambda}^{2}-1}{x_{\lambda}} \frac{m_{t}}{v}, \quad \kappa_{\eta}=\frac{m_{t}}{v} .
\end{aligned}
$$

The couplings of $\phi-Q_{1,2}-Q_{1,2}$ and $\phi-T-T(\phi=H, \eta)$ also affect the $g$ - $g$ - $\phi$ coupling through the triangle diagram at one-loop level. Here $Q_{1}$ and $Q_{2}$ are the heavy quarks for the first two generations. In both universal and anomaly-free embedding cases, the $H-Q_{1,2}-Q_{1,2}$ couplings vanish, while the $\eta-Q_{1,2}-Q_{1,2}$ couplings show a difference [4]:

$$
y_{Q_{1,2}}^{H}=0, \quad y_{Q_{1,2}}^{\eta}= \begin{cases}\frac{1}{\sqrt{2\left(t_{\beta}^{2}+x_{\lambda_{1}, \lambda_{2}}^{2}\right)}} \frac{v}{f}, & \text { for anomaly-free; } \\ -\frac{1}{\sqrt{2} t_{\beta}} \frac{v}{f}, & \text { for universal. }\end{cases}
$$

Here the $x_{\lambda_{1}, \lambda_{2}}$ are, of $\mathcal{O}(1)$, the ratios of two Yukawa couplings in the first two generations. 

$21]$

We summarize the Feynman rules for a gluon pair $\left(G^{\mu}\right.$ and $\left.G^{\nu}\right)$ with a scalar boson as

$$
\begin{aligned}
& G^{\mu}\left(k_{1}\right)-G^{\nu}\left(k_{2}\right)-H: \quad i c_{H} \delta^{a b}\left[k_{1} \cdot k_{2} \eta^{\mu \nu}-k_{1}^{\nu} k_{2}^{\mu}\right] \\
& G^{\mu}\left(k_{1}\right)-G^{\nu}\left(k_{2}\right)-\eta:-i c_{\eta} \delta^{a b} \epsilon_{\mu \nu \rho \sigma} k_{1}^{\rho} k_{2}^{\sigma}
\end{aligned}
$$

where $a$ and $b$ are the gluon color indices. The factors $c_{H}$ and $c_{\eta}$ are given by

$$
\begin{aligned}
c_{H} & =-\frac{\alpha_{s}}{4 \pi v} \sum_{f} y_{f}^{H} F_{1 / 2}^{H}\left(\tau_{f}\right), \\
c_{\eta} & =-\frac{\alpha_{s}}{4 \pi v} \sum_{f} y_{f}^{\eta} F_{1 / 2}^{\eta}\left(\tau_{f}\right),
\end{aligned}
$$

where the dimensionless loop factors of $F_{1 / 2}^{H, \eta}$ are [22]

$$
F_{1 / 2}^{H}=-2 \tau[1+(1-\tau) f(\tau)], \quad F_{1 / 2}^{\eta}=-2 \tau f(\tau)
$$

with

$$
f(\tau)= \begin{cases}{\left[\sin ^{-1}(1 / \sqrt{\tau})\right]^{2},} & \tau \geq 1 \\ -\frac{1}{4}\left[\ln \left(\eta_{+} / \eta_{-}\right)-i \pi\right]^{2}, & \tau<1\end{cases}
$$

and

$$
\tau_{f}=\frac{4 m_{f}^{2}}{m_{H}^{2}}, \quad \eta_{ \pm}=1 \pm \sqrt{1-\tau}
$$

In the limit of large $\tau$, i.e., when the particle in the loop is much heavier than $H$ or $\eta$, the loop factors approach constant values:

$$
F_{1 / 2}^{H} \rightarrow-\frac{4}{3}, \quad F_{1 / 2}^{\eta} \rightarrow-2
$$

For the process of

$$
g\left(k_{1}, \varepsilon_{1}^{\mu}\right)+g\left(k_{2}, \varepsilon_{2}^{\nu}\right) \rightarrow T\left(p_{T}\right)+\bar{t}\left(p_{T}\right)
$$

the total amplitude is

$$
M(g g \rightarrow T \bar{t})=\delta^{a b} \delta^{c d} \bar{u}_{T}\left[-g_{S}\left\{\frac{\eta_{\mu \nu}}{2}-\frac{k_{1 \mu} k_{2 \nu}}{\hat{s}}\right\} P_{L}+i g_{P} \frac{\epsilon_{\mu \nu \rho \sigma} k_{1}^{\rho} k_{2}^{\sigma}}{\hat{s}} P_{R}\right] v_{t} \varepsilon_{1}^{\mu} \varepsilon_{2}^{\nu},
$$

where $a$ and $b(c$ and $d)$ are the color indices of the gluons (of $T$ and $t$ ) and

$$
g_{S}=c_{H} \kappa_{H} \frac{\hat{s}}{\hat{s}-m_{H}^{2}}, \quad g_{P}=c_{\eta} \kappa_{\eta} \frac{\hat{s}}{\hat{s}-m_{\eta}^{2}} .
$$


The spin- and color-averaged amplitude squared is

$$
\overline{|M|^{2}}(g g \rightarrow T \bar{t})=\frac{3}{64}\left(g_{S}^{2}+g_{P}^{2}\right)\left(\hat{s}-M_{T}^{2}-m_{t}^{2}\right) \text {. }
$$

Finally, the parton level cross section is

$$
\hat{\sigma}=\frac{\lambda^{1 / 2}\left(1, M_{T}^{2} / \hat{s}, m_{t}^{2} / \hat{s}\right)}{16 \pi \hat{s}}|M|^{2} .
$$

\section{TOTAL CROSS SECTIONS AND KINEMATIC DISTRIBUTIONS}

As discussed in the Introduction, the usual evaluation of a single heavy $T$-quark production by considering only the $q b \rightarrow T q^{\prime}$ can be underestimated. The cross section of $2 \rightarrow 3$ process through $q g \rightarrow T \bar{b} q^{\prime}$ is sizable, and can in fact generate higher $p_{T}$ distributions.

In Fig. 6, we show, in the littlest Higgs model, the total cross sections of the single $T$ quark production at LHC $\left(p p \rightarrow T j\left(j^{\prime}\right) X+\bar{T} j\left(j^{\prime}\right) X\right)$ as a function of $M_{T}$ for $x_{\lambda}=1,2,3$. We have set $c=0.2$, to suppress extra contributions to EWPD, and taken $f>1.2 \mathrm{TeV}$. Note that each line starts at different $M_{T}$ since we have constrained $f$ to be above $1.2 \mathrm{TeV}$, which is marginally allowed by the EWPD. For the $2 \rightarrow 2$ production, we have included all the $t$-channel and $s$-channel sub-processes, among which the $t$-channel ones dominate. This is expected since the resonant contribution comes from the narrow window around $\hat{s} \simeq M_{W^{\prime}}^{2}$ : The total cross section is not dramatically enhanced. The total cross section for $2 \rightarrow 3$ process is smaller, but not negligible: $\sigma_{2 \rightarrow 3}$ is about $30 \sim 40 \%$ of $\sigma_{2 \rightarrow 2}$.

In Fig. [7, we show the same plots for the simplest little Higgs model. Here we take a conservative bound for $f>2 \mathrm{TeV}$, consistent with the EWPD. The minimum of $f$ also leads to the minimum of $M_{T}$ with the given $t_{\beta}$ and $x_{\lambda}$. The true minimum of $M_{T}$ occurs when $t_{\beta}=x_{\lambda}$, which we call the " $M_{T}$-minimal case". In addition, the $M_{T}^{(\min )}$ decreases with increasing $t_{\beta}$. For example, $M_{T}^{(\min )} \simeq 770 \mathrm{GeV}$ for $\left(t_{\beta}, x_{\lambda}\right)=(5,5)$, while $M_{T}^{(\min )} \simeq 560$ $\mathrm{GeV}$ for $\left(t_{\beta}, x_{\lambda}\right)=(7,7)$. Another interesting feature is that once $t_{\beta}=x_{\lambda}$, the total cross section of either $2 \rightarrow 2$ process or $2 \rightarrow 3$ process does not change much with the change of $t_{\beta}$ value, as explicitly shown in Fig. 7 . Compared with the littlest Higgs model, the $M_{T^{-}}$ minimal simplest little Higgs model has compatible total cross section with the same $M_{T}$. In the simplest little Higgs model, the total cross section for the $2 \rightarrow 3$ process is also about $30 \sim 40 \%$ of the $2 \rightarrow 2$ process. 

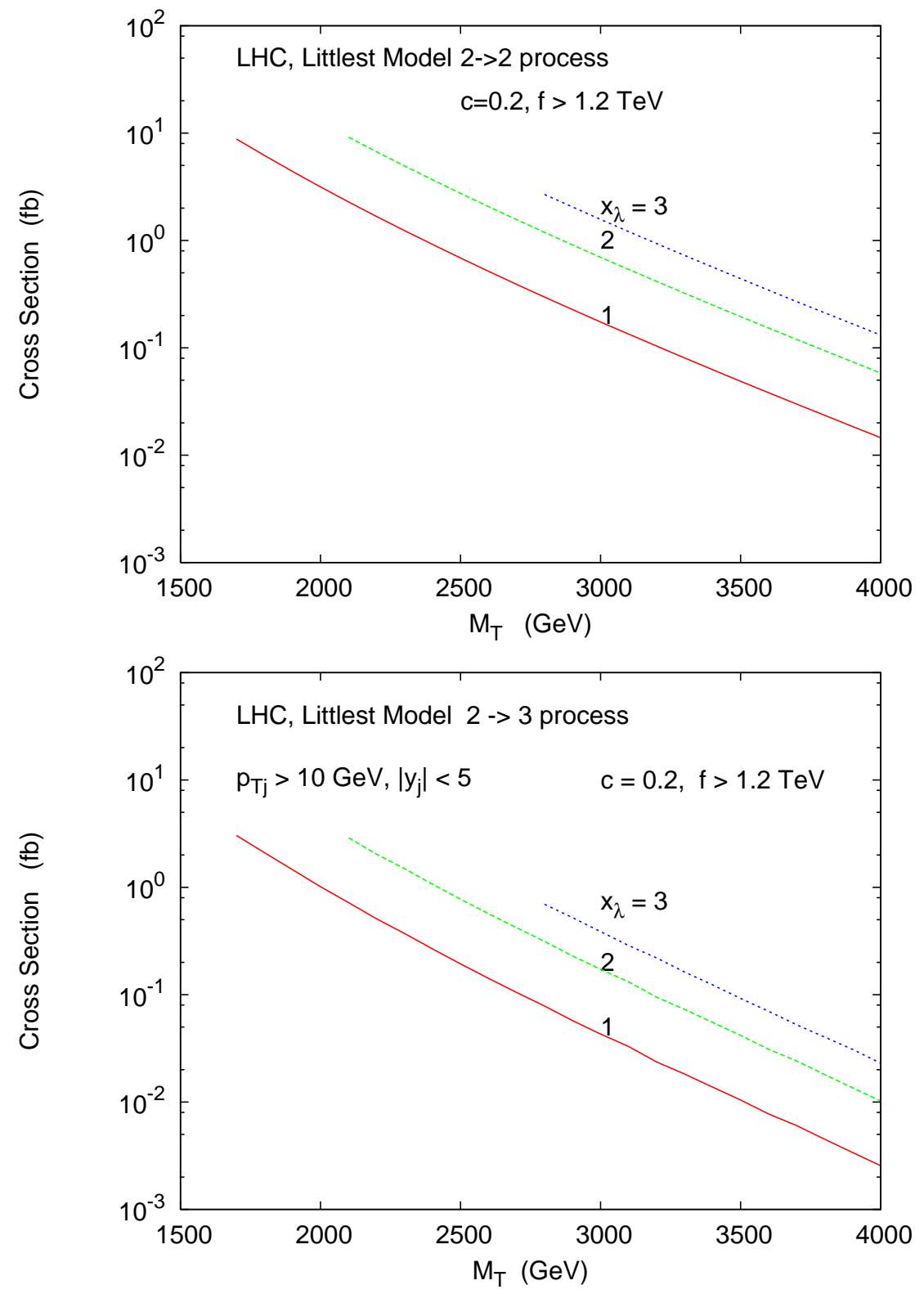

FIG. 6: Production cross section for $T$ as a function of $M_{T}$ in the littlest Higgs model. We set $c=0.2$ while taking all $f$ above $1.2 \mathrm{TeV}$. We have taken into account both $T$ and $\bar{T}$ quarks.

Figure 8 exhibits, in the littlest Higgs model, the $p_{T}$ distributions for the single $T$-quark production at LHC. The solid (red) line shows the $p_{T}$ distribution of the heavy $T$-quark in the $2 \rightarrow 2$ process, while the dashed (green) line shows the same $p_{T}$ distribution in the $2 \rightarrow 3$ process. In the $2 \rightarrow 3$ process, we also show the $p_{T}$ distributions for $b$ quark (long-dotted or blue) and for jet (dotted or pink). For the low $p_{T}$ data, which constitutes major part of the total cross section, the $2 \rightarrow 2$ process has much larger distributions compared to the 

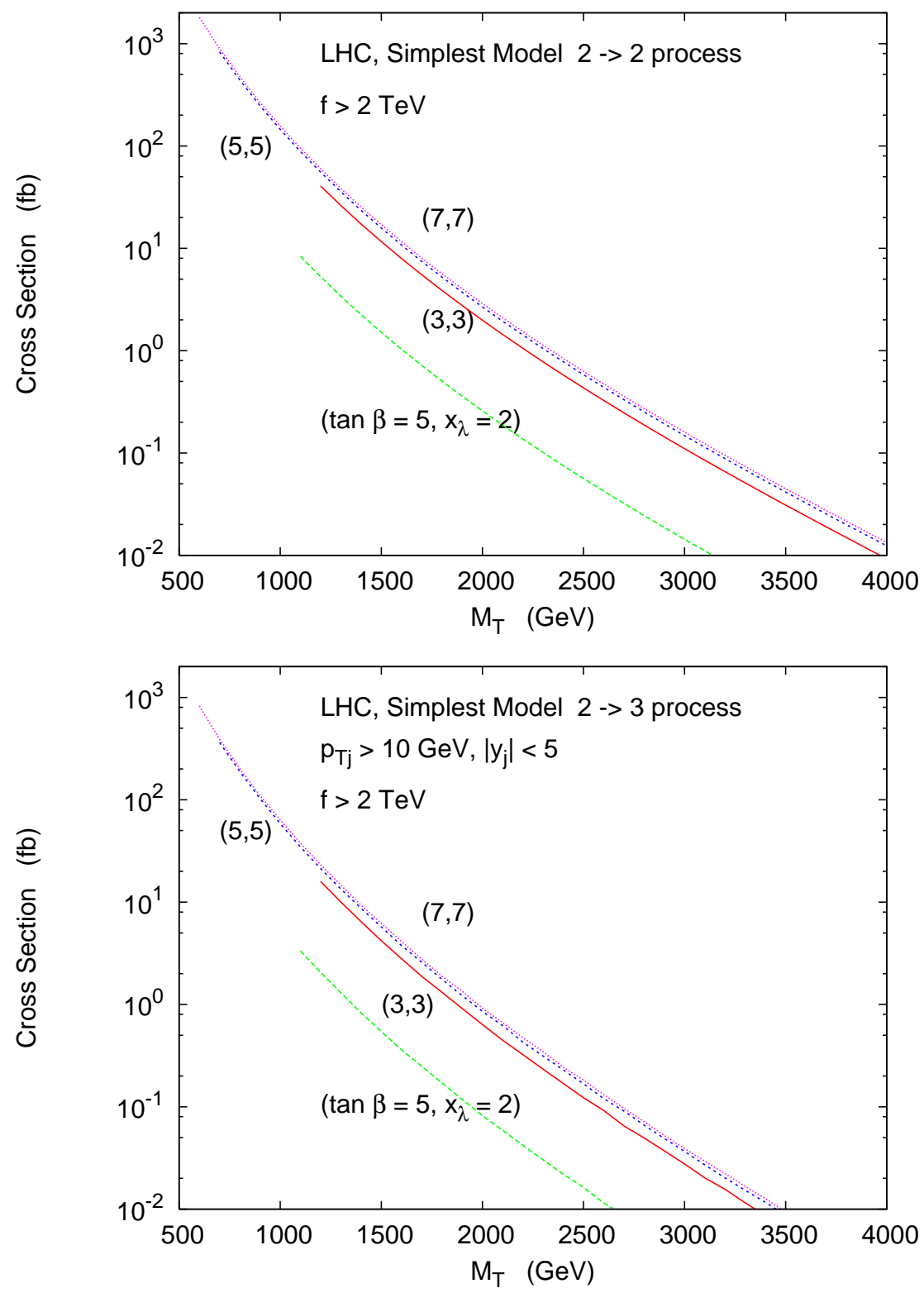

FIG. 7: Production cross section for $T$ as a function of $M_{T}$ in the simplest little Higgs model. We take $f>2 \mathrm{TeV}$ and $\left(t_{\beta}, x_{\lambda}\right)=(5,2),(3,3),(5,5)(7,7)$. We have taken into account both $T$ and $\bar{T}$ quarks.

$2 \rightarrow 3$ process. For high $p_{T}$ (above $\sim 300 \mathrm{GeV}$ ) data, however, the $2 \rightarrow 3$ process begins to dominate.

In Fig. 9. we present the same $p_{T}$ distributions in the simplest little Higgs model. Here we fix $t_{\beta}=x_{\lambda}=5$ and $M_{T}=1 \mathrm{TeV}$. The behavior of each distribution is similar to the littlest Higgs model case: In the high $p_{T}$ region, $2 \rightarrow 3$ process becomes more important. 


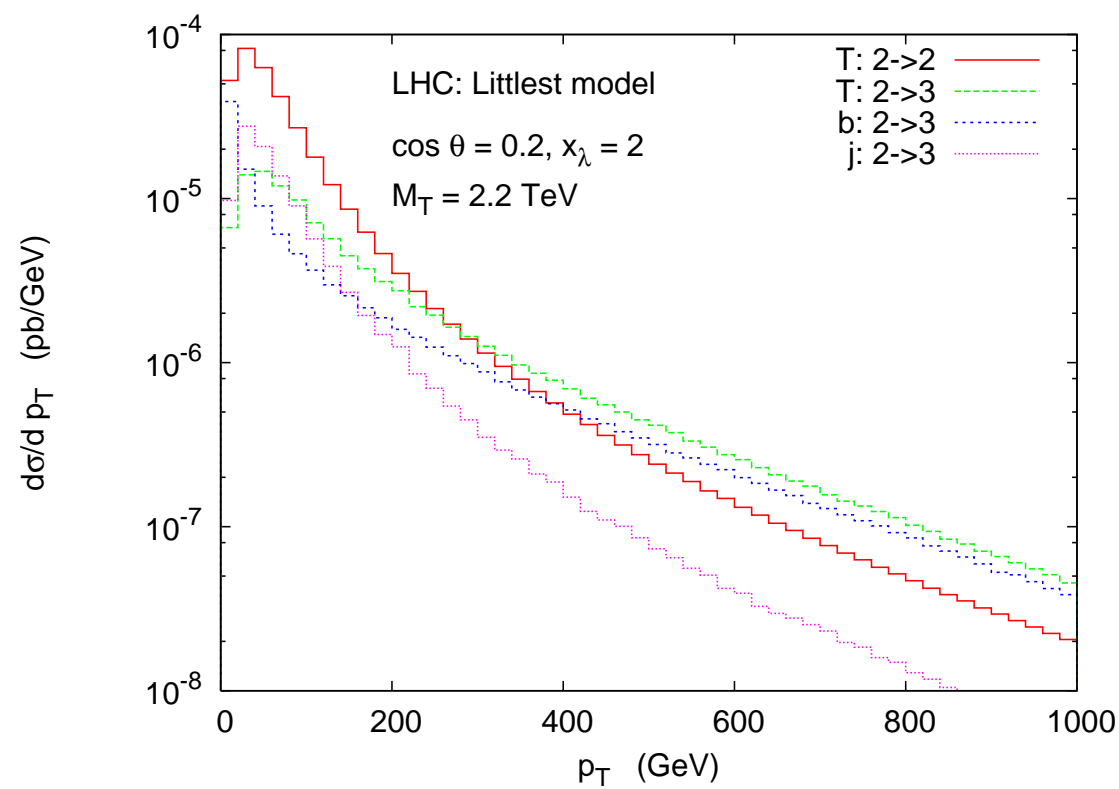

FIG. 8: $p_{T}$ distributions for the single $T$-quark production at LHC, in the littlest Higgs models.

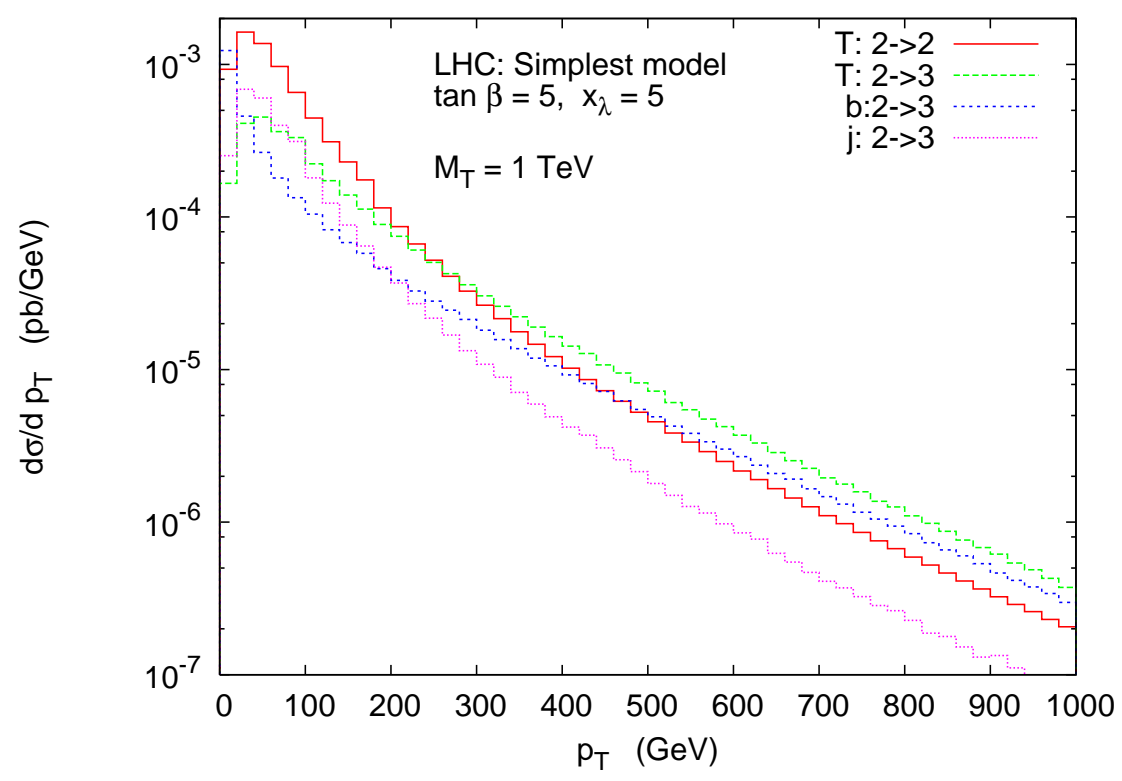

FIG. 9: $p_{T}$ distributions for the single $T$ production at LHC, in the simplest little Higgs models.

In Fig. 10, we present the rapidity distributions for out-going particles in the $2 \rightarrow 3$ process at LHC, in both the littlest and simplest little Higgs models. For the littlest Higgs model, we set $c=0.2, x_{\lambda}=2$, and $M_{T}=2.2 \mathrm{TeV}$, while for the simplest little Higgs model we set $t_{\beta}=x_{\lambda}=5$ and $M_{T}=1 \mathrm{TeV}$. We see an interestingly similar tendency: The heavy 

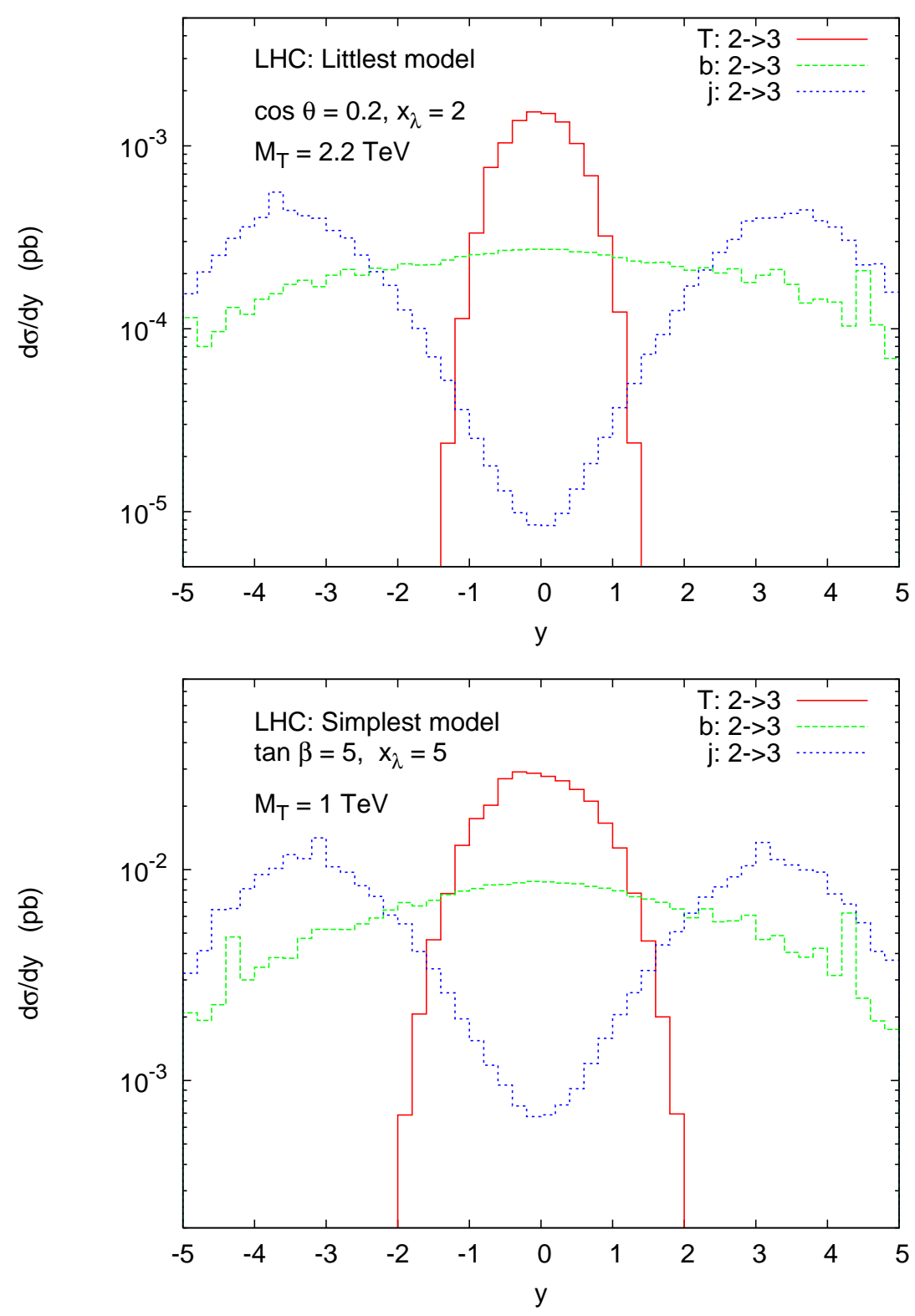

FIG. 10: $p_{T}$ distributions for the single $T$ production at LHC, in the littlest and simplest little Higgs models.

$T$-quark is likely produced in the central region, while the $b$ quark distribution is rather flat. The other light quark jet has the rapidity distribution of M-shape.

Finally we show, in Fig. 11, the total cross section of the gluon fusion production of a single $T$-quark accompanied by the SM top quark. We have fixed $t_{\beta}=x_{\lambda}=7, m_{H}=150$ $\mathrm{GeV}$, and $m_{\eta}=300 \mathrm{GeV}$. The total cross section of the gluon fusion process is $\mathcal{O}\left(10^{-3}\right)$ of the dominant cross section of the $2 \rightarrow 2$ process. In the region of small $M_{T}$ (or small $f$ ), the 


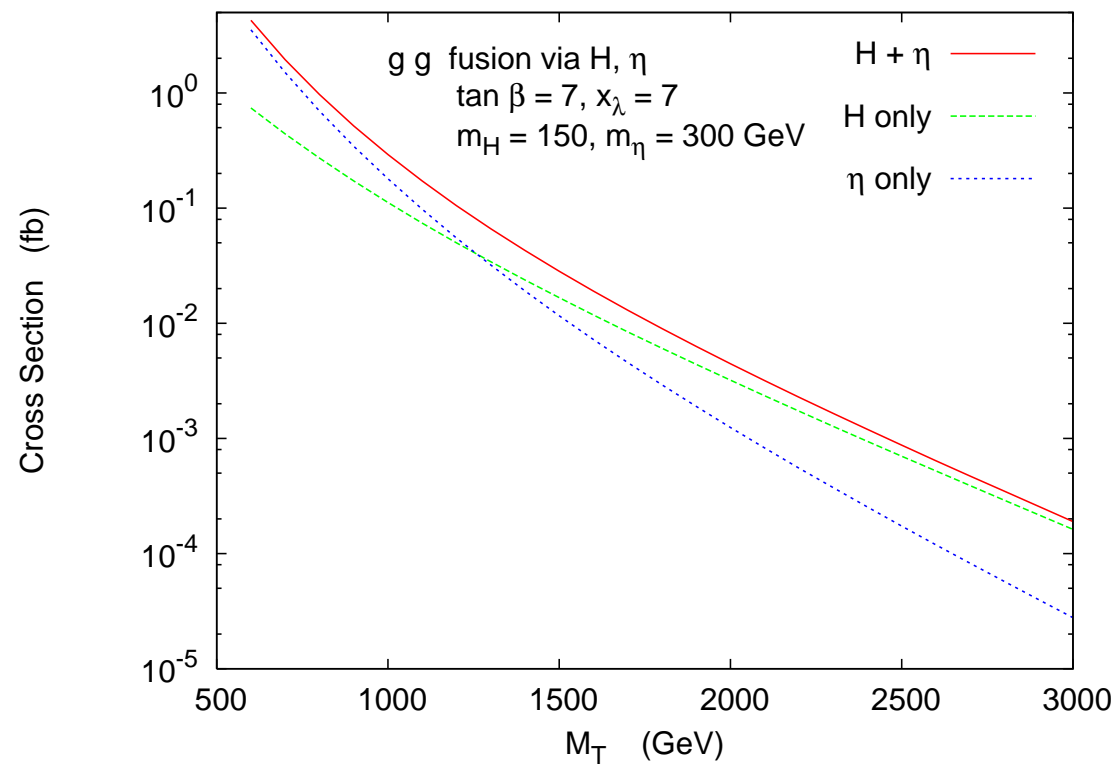

FIG. 11: In the simplest little Higgs model, total cross section of the gluon fusion production of a single $T$-quark accompanied by the SM top quark. We have taken into account both $T \bar{t}$ and $\bar{T} t$ channels.

$\eta$ contribution is dominant over the SM Higgs contribution. This is expected since the $\eta$ $t(T)-\bar{t}(\bar{T})$ couplings of $y_{t}^{\eta}\left(y_{T}^{\eta}\right)$ increase with smaller $f$ and large $t_{\beta}$, as can be seen in Eq. (38). For $M_{T} \gtrsim 1.2 \mathrm{TeV}$, the Higgs contribution becomes dominant over the $\eta$ contribution.

\section{CONCLUSIONS}

Little Higgs models can solve the little hierarchy problem that $10 \mathrm{TeV}$ cut-off in the $\mathrm{SM}$ requires a fine-tuning to the Higgs boson mass of $\mathcal{O}(100) \mathrm{GeV}$ due to the quadratic divergence of the radiative Higgs boson mass at one-loop level. In little Higgs models, the Higgs boson is a pseudo Nambu-Goldstone boson of a large global symmetry, and collective symmetry breaking mechanism prohibits the one-loop level radiative corrections to the Higgs boson mass. Phenomenologically, new heavy gauge bosons and heavy $T$-quark induce the radiative contributions which cancel the SM contributions. The heavy $T$-quark, which is colored, $S U(2)_{L}$-singlet and vector-like, is one of the most crucial ingredients, which was introduced to cancel the largest contribution of the SM top quark to the Higgs boson mass. Its production at LHC deserves a comprehensive study. In two representative little Higgs 
models, i.e., the littlest Higgs model and $S U(3)$ simplest Higgs model, we have studied the single heavy $T$-quark production without any approximation.

We have first searched for the parameter space where the heavy $T$-quark is relatively light so that it can be abundantly produced at LHC. We found that it is possible in the $S U(3)$ simplest little Higgs model. For example, the $M_{T} \simeq 560 \mathrm{GeV}$ is allowed by the EWPD when $t_{\beta}=x_{\lambda}=7$, where $t_{\beta}\left(x_{\lambda}\right)$ is the ratio of two vacuum condensates (Yukawa couplings). Moreover, the large $t_{\beta}$ is preferred by EWPD.

In literatures, only the $2 \rightarrow 2$ process of $q b \rightarrow q^{\prime} T$ has been studied for the single heavy $T$-quark production. We have extended to the $2 \rightarrow 3$ process of $q g \rightarrow q^{\prime} T \bar{b}$, the $s$-channel $q \bar{q}^{\prime} \rightarrow T \bar{b}$, and the gluon fusion process of $g g \rightarrow T \bar{t}$. The total cross section of $2 \rightarrow 3$ process is substantial, about $30 \%$ of that of $2 \rightarrow 2$ process. When we studied various kinematic distributions, we found in both little Higgs scenarios that the $2 \rightarrow 3$ process becomes more important in higher $p_{T}$ region. For $p_{T} \gtrsim 250 \mathrm{GeV}$, the $2 \rightarrow 3$ process already dominates over the $2 \rightarrow 2$ process. In the rapidity spectra, each out-going particle of the $2 \rightarrow 3$ process have distinctive features: The heavy $T$-quark is produced in the central region with $y \lesssim 1$, while the rapidity spectrum of the $b$ quark is rather flat and that of the other light quark-jet is of M-shape. We have also considered the $s$-channel process of $q \bar{q}^{\prime} \rightarrow T \bar{b}$, which is expected to be sizable if the resonant decay of the heavy charged gauge boson $W^{\prime}$ is possible. We examined the parameter space for $M_{W^{\prime}}>M_{T}$, which was found to be consistent with the EWPD. Unfortunately, the total cross section is too suppressed due to a very small region of the resonant decay.

Finally, we have taken into account of the gluon fusion production of the heavy $T$-quark and the SM top quark, which is mediated by the Higgs boson and the pseudo-scalar $\eta$. This can be substantial when the heavy $T$-quark is light enough. However, we found that the total cross section is still suppressed, only $\mathcal{O}\left(10^{-3}\right)$ of the dominant $2 \rightarrow 2$ process. An interesting feature is that the $\eta$ contribution is dominant over the Higgs contribution for relatively light $M_{T}$.

\section{Acknowledgments}

The work of C.S.K. was supported in part by CHEP-SRC Program and in part by the KRF Grant funded by the Korean Government (MOEHRD) No. KRF-2005-070-C00030. 
The work of JS is supported by KRF under grant No. R04-2004-000-10164-0. The work of KYL is supported by Korea Research Foundation Grant (KRF-2003-050-C00003). The work of KC is supported by the National Science Council of Taiwan under grant no. 942112-M-007-010- and 95-2112-M-007-001-.

\section{APPENDIX A: HELICITY AMPLITUDES AND KINEMATICS FOR $q g \rightarrow q^{\prime} \bar{b} T$}

For the process of

$$
u\left(p_{1}\right)+g\left(p_{2}\right) \rightarrow d\left(k_{1}\right)+T\left(k_{2}\right)+\bar{b}\left(k_{3}\right),
$$

the amplitude-squared (after summing over the colors and helicities and averaging over initial colors and helicities) is

$$
\begin{gathered}
\overline{|M|^{2}}=\frac{384}{4 \cdot 24} g_{s}^{2} \\
\times\left\{\begin{array}{r}
\left|Q_{p 1 k 1}\right|^{2}\left[2 D_{k 2 p 2}^{2}\left\langle k_{3} p_{1}\right\rangle\left\{\left\langle k_{1} p_{2}\right\rangle\left\langle k_{2} p_{2}\right\rangle+M_{T}^{2}\left\langle k_{1} p_{2}\right\rangle-M_{T}^{2}\left\langle k_{1} k_{2}\right\rangle\right\}\right. \\
+D_{p 2 k 3}^{2}\left\langle k_{1} k_{2}\right\rangle\left\{-2 m_{b}^{2}\left\langle k_{3} p_{1}\right\rangle+\hat{s}\left\langle k_{3} p_{2}\right\rangle+\hat{s} m_{b}^{2}\right\} \\
+D_{p 2 k 3} D_{k 2 p 2}\left\{2\left\langle k_{1} k_{3}\right\rangle\left\langle k_{2} p_{2}\right\rangle\left\langle k_{3} p_{1}\right\rangle-2\left\langle k_{1} k_{2}\right\rangle\left\langle k_{3} p_{1}\right\rangle\left\langle k_{3} p_{2}\right\rangle+4\left\langle k_{1} k_{2}\right\rangle\left\langle k_{2} k_{3}\right\rangle\left\langle k_{3} p_{1}\right\rangle\right. \\
-2\left\langle k_{1} k_{2}\right\rangle\left\langle k_{2} p_{2}\right\rangle\left\langle k_{3} p_{1}\right\rangle+2\left\langle k_{1} k_{2}\right\rangle\left\langle k_{2} p_{1}\right\rangle\left\langle k_{3} p_{2}\right\rangle-2\left\langle k_{1} p_{2}\right\rangle\left\langle k_{2} k_{3}\right\rangle\left\langle k_{3} p_{1}\right\rangle \\
\left.\left.-\hat{s}\left\langle k_{1} k_{2}\right\rangle\left\langle k_{2} k_{3}\right\rangle\right\}\right] \\
+\left|Q_{k 2 k 3}\right|^{2}\left[2 D_{k 1 p 2}^{2}\left\langle k_{1} p_{2}\right\rangle\left\langle k_{2} p_{2}\right\rangle\left\langle k_{3}\right\rangle p_{1}+D_{p 1 p 2}^{2} s\left\langle k_{1} k_{2}\right\rangle\left\langle k_{3} p_{2}\right\rangle\right. \\
+D_{k 1 p 2} D_{p 1 p 2}\left\{-2\left\langle k_{1} p_{2}\right\rangle\left\langle k_{2} p_{1}\right\rangle\left\langle k_{3} p_{1}\right\rangle-2\left\langle k_{1} p_{2}\right\rangle\left\langle k_{1} k_{2}\right\rangle\left\langle k_{3} p_{1}\right\rangle+2\left\langle k_{1} p_{1}\right\rangle\left\langle k_{2} p_{2}\right\rangle\left\langle k_{3} p_{1}\right\rangle\right. \\
\quad-2\left\langle k_{1} p_{1}\right\rangle\left\langle k_{1} k_{2}\right\rangle\left\langle k_{3} p_{2}\right\rangle-4\left\langle k_{1} p_{1}\right\rangle\left\langle k_{1} k_{2}\right\rangle\left\langle k_{3} p_{1}\right\rangle \\
\left.\left.\left.+\hat{s}\left\langle k_{1} k_{2}\right\rangle\left\langle k_{3} p_{1}\right\rangle+\hat{s}\left\langle k_{1} k_{2}\right\rangle\left\langle k_{1} k_{3}\right\rangle\right\}\right]\right\}
\end{array}\right. \\
\quad(\mathrm{A} 3)
\end{gathered}
$$

where $\left\langle p_{i} p_{j}\right\rangle \equiv p_{i} \cdot p_{j}$, and the propagator factors are

$$
\begin{aligned}
\hat{s} & =\left(p_{1}+p_{2}\right)^{2}, \quad D_{k 1 p 2}=\frac{1}{\left(k_{1}-p_{2}\right)^{2}} \\
D_{p 1 p 2} & =\frac{1}{\left(p_{1}+p_{2}\right)^{2}}, \quad D_{k 2 p 2}=\frac{1}{\left(k_{2}-p_{2}\right)^{2}-m_{T}^{2}}, \quad D_{p 2 k 3}=\frac{1}{\left(p_{2}-k_{3}\right)^{2}-m_{b}^{2}}, \\
Q_{p 1 k 1} & =\frac{g_{u d}^{W} g_{T b}^{W}}{\left(p_{1}-k_{1}\right)^{2}-m_{w}^{2}}+\frac{g_{u d}^{W^{\prime}} g_{T b}^{W^{\prime}}}{\left(p_{1}-k_{1}\right)^{2}-M_{W^{\prime}}^{2}}, \\
Q_{k 2 k 3} & =\frac{g_{u d}^{W} g_{T b}^{W}}{\left(k_{2}+k_{3}\right)^{2}-m_{w}^{2}}+\frac{g_{u d}^{W^{\prime}} g_{T b}^{W^{\prime}}}{\left(k_{2}+k_{3}\right)^{2}-M_{W^{\prime}}^{2}} .
\end{aligned}
$$


In order to specify the kinematic variable we define the energies of involving particles, in the parton c.m. frame, by

$$
\begin{aligned}
& E_{u}=\frac{\sqrt{\hat{s}}}{2}, \quad E_{g}=\frac{\sqrt{\hat{s}}}{2} \\
& E_{d}=\frac{\sqrt{\hat{s}}}{2}(1-\eta), \quad E_{\bar{b}}=\frac{\sqrt{\hat{s}}}{2}(1-\zeta), \quad E_{T}=\frac{\sqrt{\hat{s}}}{2}(\eta+\zeta) .
\end{aligned}
$$

Then the final three momenta $\left(\vec{p}_{d}, \overrightarrow{p_{\bar{b}}}, \overrightarrow{p_{T}}\right)$ lie in a plane. We define the angle $\theta$ by

$$
\cos \theta=-\hat{p}_{d} \cdot \hat{p}_{\bar{b}}=1-2 \frac{\eta \zeta-M_{T}^{2} / \hat{s}}{(1-\eta)(1-\zeta)} .
$$

Kinematic boundaries for $\cos \theta= \pm 1$ lead to the allowed parameter space of

$$
\eta \zeta \geq \frac{M_{T}^{2}}{\hat{s}}, \quad \eta+\zeta \leq 1+\mu_{T} .
$$

And $\alpha \in[0,2 \pi]$ and $\cos \beta \in[0,1]$.

[1] N. Arkani-Hamed, A. G. Cohen and H. Georgi, Phys. Lett. B 513, 232 (2001); N. ArkaniHamed, A. G. Cohen, E. Katz, A. E. Nelson, T. Gregoire and J. G. Wacker, JHEP 0208, 021 (2002).

[2] H. Georgi and A. Pais, Phys. Rev. D 10, 539 (1974).

[3] I. Low, W. Skiba and D. Smith, Phys. Rev. D 66, 072001 (2002); S. Chang and J. G. Wacker, Phys. Rev. D 69, 035002 (2004); W. Skiba and J. Terning, Phys. Rev. D 68, 075001 (2003); S. Chang, JHEP 0312, 057 (2003); E. Katz, J. y. Lee, A. E. Nelson and D. G. E. Walker, JHEP 0510, 088 (2005); I. Low, JHEP 0410, 067 (2004); H. C. Cheng and I. Low, JHEP 0408, 061 (2004).

[4] T. Han, H. E. Logan and L. T. Wang, JHEP 0601, 099 (2006).

[5] G. Burdman, M. Perelstein and A. Pierce, Phys. Rev. Lett. 90, 241802 (2003) [Erratum-ibid. 92, 049903 (2004)]; J. Hubisz and P. Meade, Phys. Rev. D 71, 035016 (2005); S. C. Park and J. Song, Phys. Rev. D 69, 115010 (2004).

[6] G. Azuelos et al., Eur. Phys. J. C 39S2, 13 (2005)

[7] M. Perelstein, M. E. Peskin and A. Pierce, Phys. Rev. D 69, 075002 (2004);

[8] M. Perelstein, arXiv:hep-ph/0512128.

[9] G. Azuelos et al., Eur. Phys. J. C 39S2, 13 (2005) arXiv:hep-ph/0402037. 
[10] N. Arkani-Hamed, A. G. Cohen, E. Katz and A. E. Nelson, JHEP 0207, 034 (2002).

[11] T. Han, H. E. Logan, B. McElrath and L. T. Wang, Phys. Rev. D 67, 095004 (2003).

[12] C. Csaki, J. Hubisz, G. D. Kribs, P. Meade and J. Terning, Phys. Rev. D 67, 115002 (2003); J. L. Hewett, F. J. Petriello and T. G. Rizzo, JHEP 0310, 062 (2003); M. C. Chen and S. Dawson, Phys. Rev. D 70, 015003 (2004); W. Kilian and J. Reuter, Phys. Rev. D 70, $015004(2004)$.

[13] C. Csaki, J. Hubisz, G. D. Kribs, P. Meade and J. Terning, Phys. Rev. D 68, 035009 (2003).

[14] J. A. Casas, J. R. Espinosa and I. Hidalgo, JHEP 0503, 038 (2005).

[15] M. Schmaltz, JHEP 0408, 056 (2004).

[16] Z. Han and W. Skiba, Phys. Rev. D 72, 035005 (2005).

[17] D. E. Kaplan and M. Schmaltz, JHEP 0310, 039 (2003);

[18] O. C. W. Kong, arXiv:hep-ph/0307250, O. C. W. Kong, J. Korean Phys. Soc. 45, S404 (2004).

[19] T. Stelzer, Z. Sullivan and S. Willenbrock, Phys. Rev. D 56, 5919 (1997).

[20] W. Kilian, D. Rainwater and J. Reuter, Phys. Rev. D 71, 015008 (2005).

[21] S. Y. Choi, K. Hagiwara and J. S. Lee, Phys. Lett. B 529, 212 (2002).

[22] M. A. Shifman, A. I. Vainshtein, M. B. Voloshin and V. I. Zakharov, Sov. J. Nucl. Phys. 30, 711 (1979) [Yad. Fiz. 30, 1368 (1979)]; L.b. Okun, Leptons and Quarks (North-holland, Amsterdam, 1982). 